Ergod. Th. \& Dynam. Sys. (1984), 4, 405-420

Printed in Great Britain

\title{
On the notion of the dimension with respect to a dynamical system
}

\author{
YA. B. PESIN \\ All-Union Extra-Mural Construction Engineering Institute, \\ Sredne Kalitnikovskaja St., 30, Moscow, 109807, USSR
}

(Received 17 December 1982 and revised 30 October 1983)

\begin{abstract}
For the invariant sets of dynamical systems a new notion of dimension - the so-called dimension with respect to a dynamical system - is introduced. It has some common features with the general topological notion of the dimension, but it also reflects the dynamical properties of the system. In the one-dimensional case it coincides with the Hausdorff dimension. For multi-dimensional hyperbolic sets formulae for the calculation of our dimension are obtained. These results are generalizations of Manning's results obtained by him for the Hausdorff dimension in the two-dimensional case.
\end{abstract}

\section{Introduction}

We consider a diffeomorphism $f$ of a smooth Riemannian manifold $M$, and an $f$-invariant set $\Lambda$, consisting of the trajectories which are more or less unstable. This instability can be characterized in many ways in particular by means of the absence of zero Lyapunov exponents. It often implies some stochastic properties of the map $f$ on the set $\Lambda$, for example, positivity of entropy with respect to a suitable measure with 'good' properties. On the other hand the unstable invariant set has, as a rule, a complicated geometric structure and can be characterized from this point of view by means of its Hausdorff dimension. The unstable invariant sets arise both in dissipative and conservative systems. In the first case we have so-called strange attractors (cf. [14]). In the second case the situation is similar to that which arises in the neighbourhoods of hyperbolic sets. The establishing of connections between instability, stochastic properties and topological structure of unstable invariant sets is one of the fundamental problems of the hyperbolic theory. Any relationship between Lyapunov exponents, entropy and Hausdorff dimension can be considered as a reflection of this connection. Moreover using such a relationship one can calculate one of these characteristics from the others. In particular one can hope to compute Hausdorff dimension in the case when $\Lambda$ is a strange attractor. This fact can play an important role in the study of the turbulence problem as Takens pointed out recently (cf. [16]).

Some relationships between Lyapunov exponents, entropy and Hausdorff dimension were obtained in the two-dimensional case for basic sets of $A$ diffeomorphisms (cf. [11], [12]). 
In other cases and especially in multi-dimensional ones the various estimates of Hausdorff dimension from below and from above were obtained, for example, in the papers [6], [10], [17]. However the exact expressions for the Hausdorff dimension in terms of Lyapunov exponents were not found. It seems to us that this is not by chance. There are arguments showing that Hausdorff dimension does not adequately reflect dynamical properties of dynamical systems. At least it cannot be expressed by Lyapunov exponents only (cf. remarks in [9] in this connection). To illustrate this fact we consider the three-dimensional Smale's horseshoe constructed from the three-dimensional cube by means of the following procedure:

(1) expansion in some direction with coefficient $\gamma$;

(2) contraction in two other directions with coefficients $\lambda, \mu$;

(3) bending and laying on the initial cube.

It is easy to show that the Hausdorff dimension of this horseshoe depends not only on the numbers $\gamma, \lambda, \mu$ but also on the number of bendings.

In the present paper we introduce a new notion of dimension which we call 'the dimension with respect to a map'. This notion has some common features with the general topological notion of dimension (cf. [8]). It coincides with Hausdorff dimension in some cases (in particular for subsets of the straight line). However it seems that this notion is better adapted to the description of dynamical properties of systems than Hausdorff dimension. In particular, we extend the results of [11], [12] to the multi-dimensional case. Moreover at present in the investigation of concrete physical models in which the notion of dimension is used there are no reasons why Hausdorff dimension is preferable to our dimension. Finally the simple formulae for the dimension with respect to a map which we obtain in the present paper allow us to compute this dimension in each concrete case easily.

Recently V. Afraimovich and the author obtained the estimates from below and from above for the Hausdorff dimension of the hyperbolic set arising in the neighbourhood of a homoclinic orbit (cf. [1]). Only the two-dimensional case is considered in [1]; however the results and their proofs given there can easily be generalized to the multi-dimensional case if we replace Hausdorff dimension by our new dimension.

Recall the definition of Hausdorff dimension (for example, cf. [2]). Let $X$ be a metric space. The Hausdorff measure of $X$ is defined by the formula:

$$
\begin{gathered}
m_{\lambda}(X)=\lim _{\varepsilon \rightarrow 0} \inf _{\left\{U_{s}\right\}_{s \in S}}\left\{\sum_{s \in S}\left(\operatorname{diam} U_{s}\right)^{\lambda}:\left\{U_{s}\right\} \text { is an open covering of } X\right. \\
\text { and diam } \left.U_{s} \leq \varepsilon\right\} .
\end{gathered}
$$

It is easy to show that there exists a real number $\lambda_{0}$ such that $m_{\lambda}(X)=\infty$ for any $\lambda<\lambda_{0}$ and $m_{\lambda}(X)=0$ for any $\lambda>\lambda_{0}$. The number $\lambda_{0}$ is called the Hausdorff dimension of $X$. Thus we have

$$
\operatorname{dim}_{H} X=\inf \left\{\lambda: m_{\lambda}(X)=0\right\}=\sup \left\{\lambda: m_{\lambda}(X)=\infty\right\} .
$$

Suppose that $X$ is a Borel subset of a smooth manifold $M$ and $p=\operatorname{dim} M$. It is easy to see that in order to calculate Hausdorff dimension of $X$ one can consider only the coverings consisting of open balls. Therefore one can rewrite the expression 
for the Hausdorff measure of $X$ in the form

$$
m_{\lambda}(X)=\lim _{\varepsilon \rightarrow 0} \inf _{\left\{U_{s}\right\}_{s \in S}}\left\{\sum_{s \in S}\left[V\left(U_{s}\right)\right]^{\lambda / p}\right\}
$$

where $U_{s}$ is an open ball in $M$ with its centre at $x \in X$ and radius $\leq \varepsilon, \bigcup_{s \in S} U_{s} \supset X$. Here $V\left(U_{s}\right)$ is the $p$-dimensional volume of the ball $U_{s}$. We can get new measures of $X$ in $M$ if we take in the formula (1) some other systems of open sets in $M$, satisfying certain 'reasonable' conditions, instead of the system of open balls. Every such measure induces the corresponding notion of dimension of $X$ which has some common features with a general topological notion of dimension. Of course our notion of dimension is not a purely topological one because it depends on the manifold $M$ and on the choice of the system of open sets.

Now we consider the following situation: $N$ is a smooth manifold, $f: N \rightarrow N$ is a diffeomorphism, $M \subset N$ is a smooth submanifold, $X \subset M$ is a Borel subset. ihe map $f$ induces (under suitable conditions) the special system of open sets in $M$ and the corresponding notion of dimension is just the dimension with respect to the map $f$.

\section{Definitions and formulation of results}

I. After these preliminary considerations we will give explicit formulations. Let $M$ be a smooth Riemannian manifold, $\nu$ be the Riemannian volume in $M, X \subset M$ be a Borel set. Let $\bar{U}=\left\{U_{s}\right\}_{s \in S}$ be a system of open subsets in $M$ having the following property (with respect to $X$ ):

(P1) for any $\varepsilon>0$, there exists a subsystem $\bar{V}=\left\{V_{i}\right\}_{i \in I}$ such that $V_{i} \in \bar{U}$, diam $V_{i} \leq \varepsilon$ and $\bigcup_{i \in I} V_{i} \supset X$.

If $Y \subset X$ and $\bar{U}$ is the system of open subsets having the property (P1) with respect to $X$ then it obviously has the property (P1) with respect to $Y$.

Let $Y \subset X$ be a subset and $\alpha \in(0,1)$. Write

$$
M_{\alpha, \varepsilon}(Y, X, M, \bar{U})=\inf _{\bar{v} \subset \bar{U}}\left\{\sum_{i \in I} \nu\left(V_{i}\right)^{\alpha}: \bar{V}=\left\{V_{i}\right\}_{i \in I}, \operatorname{diam} V_{i} \leq \varepsilon, \bigcup_{i \in I} V_{i} \supset Y\right\} .
$$

For the sake of brevity we will sometimes denote $M_{\alpha, \varepsilon}(Y, X, M, \bar{U})$ by $\left(M_{\alpha, \varepsilon}(Y)\right.$. It is obvious that the function $M_{\alpha, \varepsilon}(Y)$ increases as $\varepsilon \rightarrow 0$. Thus we may define the limit

$$
m_{\alpha}(Y) \stackrel{\text { def }}{=} m_{\alpha}(Y, X, M, \bar{U}) \stackrel{\text { def }}{=} \lim _{\varepsilon \rightarrow 0} M_{\alpha, \varepsilon}(Y, X, M, \bar{U})
$$

The function $m_{\alpha}(Y)$ is the outer measure on the space of all subsets of $X$. Namely:

(1) if $Y_{1} \subset Y_{2} \subset X$, then $m_{\alpha}\left(Y_{1}\right) \leq m_{\alpha}\left(Y_{2}\right)$;

(2) if $Y=\bigcup_{n} Y_{n}$, and $Y \subset X$ then

$$
m_{\alpha}(Y) \leq \sum_{n} m_{\alpha}\left(Y_{n}\right)
$$

(3) $m_{\alpha}(\varnothing)=0$. 
The first property is obvious and means that $m_{\alpha}(Y)$ is a monotone function of $Y$. The third property is also obvious. The second property is proved as in [2]. The inequality (2) expresses that fact that the function $m_{\alpha}(Y)$ is $\sigma$-semiadditive.

One can show that every Borel set in $X$ is measurable with respect to the outer measure $m_{\alpha}(Y)$, (and $m_{\alpha}$ is a $\sigma$-additive measure on the $\sigma$-algebra of all Borel subsets in $X$ ). In addition $m_{\alpha}$ is a metric and a regular measure (cf. [7]). It is easy to see that there exists $\alpha_{0}$ such that $m_{\alpha}(Y)=\infty$ for any $\alpha<\alpha_{0}$ and $m_{\alpha}(Y)=0$ for any $\alpha>\alpha_{0}$. We call the number

$$
\begin{aligned}
\operatorname{dim}_{(X, M, \bar{U})} Y & =p \sup \left\{\alpha: m_{\alpha}(Y)=\infty\right\} \\
& =p \inf \left\{\alpha: m_{\alpha}(Y)=0\right\}=p \alpha_{0}
\end{aligned}
$$

the dimension of $Y$ with respect to the triplet $(X, M, \bar{U})$, (recall that $p=\operatorname{dim} M)$. The following assertions are obvious and justify the term dimension.

Proposition 1. (1) If $\bar{U}=\left\{B_{M}(x, \varepsilon), x \in X, \varepsilon>0\right\}$, then $\operatorname{dim}_{(X, M, \bar{U})} Y=\operatorname{dim}_{H} Y$, (here $\left.B_{M}(x, \varepsilon)\right)$ is the ball in $M$ with centre at $x$ and radius $\left.\varepsilon\right)$.

(2) If $p=\operatorname{dim} M=1$ then $\operatorname{dim}_{(X, M, \bar{U})} Y=\operatorname{dim}_{H} Y$ for any system $\bar{U}$ having the property ( $\mathrm{P} 1)$ with respect to $X$ and consisting of connected sets.

(3) If $\bar{U}_{1}, \bar{U}_{2}$ are two systems of open sets having the property (P1) with respect to $X$ and $\bar{U}_{1} \subset \bar{U}_{2}$ then

$$
\operatorname{dim}_{\left(X, M, \bar{U}_{1}\right)} Y \geq \operatorname{dim}_{\left(X, M, \bar{U}_{2}\right)} Y .
$$

(4) If $Y_{1} \subset Y_{2} \subset X$, and the system $\bar{U}$ has the property (P1) with respect to $X$ then

$$
\operatorname{dim}_{(X, M, \bar{U})} Y_{1} \leq \operatorname{dim}_{(X, M, \bar{U})} Y_{2} \text {. }
$$

(5) If $Y_{n} \subset X(n \in \mathbb{Z}), Y=\bigcup_{n} Y_{n} \subset X$ and the system $\bar{U}$ has the property (P1) with respect to $X$ then we have

(the proof follows easily from [2]).

$$
\operatorname{dim}_{(X, M, \bar{U})} Y=\sup _{n}\left\{\operatorname{dim}_{(X, M, \bar{U})} Y_{n}\right\},
$$

(6) If $X$ is an open subset in $M$, then for any system having the property (P1) with respect to $X$,

$$
\operatorname{dim} M=\operatorname{dim}_{(X, M, \bar{U})} X=\operatorname{dim} X .
$$

(7) If $X$ is finite or countable then for any system of open sets in $M$ having the property $(\mathrm{Pl})$ with respect to $X$ we have

$$
\operatorname{dim}_{(X, M, \bar{U})} X=0 .
$$

The number $\operatorname{dim}_{(X, M, \bar{U})} Y$ does not depend on the Riemannian metric of the manifold $M$, because any two Riemannian metrics on $M$ are equivalent.

Let $\bar{U}^{i}=\left\{U_{s}^{i}, s \in S\right\}$ be a system of open sets in the manifold $M_{i}$ having the property (Pl) with respect to the Borel set $X_{i} \subset M_{i}, i=1,2$ and $\operatorname{dim} M_{1}=\operatorname{dim} M_{2}$. The systems $\bar{U}^{1}$ and $\bar{U}^{2}$ are called equivalent if there exists $C>0$ and a one-to-one map $\Psi: \bar{U}^{1} \rightarrow \bar{U}^{2}$ such that:

(1) $C^{-1} \nu\left(U_{s}^{1}\right) \leq \nu\left(\Psi\left(U_{s}^{1}\right)\right) \leq C \nu\left(U_{s}^{1}\right), \quad s \in S$;

(2) if the system $\left\{U_{s_{i}}^{1}\right\}$ covers the set $X_{1}$ then the system $\left\{\Psi\left(U_{s,}^{1}\right)\right\}$ covers the set $X_{2}$

(3) if the system $\left\{U_{s_{i}}^{2}\right\}$ covers the set $X_{2}$ then the system $\left\{\Psi^{-1}\left(U_{s_{i}}^{2}\right)\right\}$ covers the set $X_{1}$. 
It is not difficult to prove the following statement.

Proposition 2. $\operatorname{dim}_{\left(X_{1}, M_{1}, \bar{U}^{1}\right)} X_{1}=\operatorname{dim}_{\left(X_{2}, M_{2}, \bar{U}^{2}\right)} X_{2}$.

II. Let $N$ be a smooth Riemannian manifold, $f: N \rightarrow N$ be a diffeomorphism, $M \subset N$ be a smooth submanifold, $X \subset M$ be a Borel set. Fix $\delta>0$ and consider the system of open sets

$\bar{U}_{f, \delta}(X)=\left\{U \subset M\right.$ : there exist $x \in X$ and $n \in \mathbb{Z}$ such that $U=U_{|n|}$ where

$$
U_{1}=B_{f^{n}(M)}\left(f^{n}(x), \delta\right) \text {, }
$$

and for $n \geq 0$

$$
U_{k}=f^{-1}\left(U_{k-1}\right) \cap B_{f^{n-k}(M)}\left(f^{n-k}(x), \delta\right)
$$

but for $n \leq 0$

$$
\left.U_{k}=f\left(U_{k-1}\right) \cap B_{f^{n+k}(M)}\left(f^{n+k}(x), \delta\right)\right\} .
$$

Suppose that for all sufficiently small $\delta>0$ the systems $\bar{U}_{f, \delta}$ have the property (Pl) with respect to $X$. Let $Y \subset X$ be a subset. Consider the outer measures $m_{\alpha}\left(Y, X, M, \bar{U}_{f, \delta}(X)\right)$ and assume that the following condition holds

(P2) there exists $\delta_{0}>0$ such that for any $\delta_{1}, \delta_{2} \in\left(0, \delta_{0}\right]$ we have

$$
\begin{aligned}
C_{1} m_{\alpha}\left(Y, X, M, \vec{U}_{f, \delta_{2}}(X)\right) & \leq m_{\alpha}\left(Y, X, M, \bar{U}_{f, \delta_{1}}(X)\right) \\
& \leq C_{2} m_{\alpha}\left(Y, X, M, \bar{U}_{f, \delta_{2}}(X)\right),
\end{aligned}
$$

where $C_{i}=C_{i}\left(\delta_{1}, \delta_{2}\right)>0, i=1,2$ are constants. It follows from the condition (P2) that for any $\delta \in\left(0, \delta_{0}\right]$

$$
\operatorname{dim}\left(X, M, \bar{U}_{f, \delta}(X)\right) Y=\text { const. }
$$

This common value is called the dimension of $Y$ with respect to the map $f$ (and also with respect to $X$ and $M$ ) and it is denoted by $\operatorname{dim}_{f} Y$, (sometimes when we wish to emphasize the dependence on $M$ we use the notation $\operatorname{dim}_{(M, f)} Y$ ). We note that the dimension with respect to a map depends in general on the choice of the Riemannian metric on $M$. The following assertion is a consequence of proposition 1 and the definitions given above.

Proposition 3. Suppose that the systems $\bar{U}_{f, \delta}(X)$ have the properties (P1), (P2). Then

(1) if $p=\operatorname{dim} M=1$, then $\operatorname{dim}_{f} Y=\operatorname{dim}_{H} Y, \quad(Y \subset X)$;

(2) if $Y_{1} \subset Y_{2} \subset X$, then $\operatorname{dim}_{f} Y_{1} \leq \operatorname{dim}_{f} Y_{2}$;

(3) if $Y_{n} \subset X(n \in \mathbb{Z})$ and $Y=\bigcup_{n} Y_{n} \subset X$ then

$$
\operatorname{dim}_{f} Y=\sup _{n} \operatorname{dim}_{f} Y_{n}
$$

(4) if $X$, is an open subset in $M$ then

$$
\operatorname{dim} M=\operatorname{dim}_{f} X=\operatorname{dim} X
$$

(5) if $X$ is finite or countable then $\operatorname{dim}_{f} X=0$;

(6) $\operatorname{dim}_{f} Y=\operatorname{dim}_{f^{-1}} Y$ for any $Y \subset X$.

During a discussion of this paper with D. V. Anosov he drew my attention to an interesting example illustrating the distinction between Hausdorff dimension and 
our dimension with respect to a map. Let $N=T^{2} \times T^{2}$ be the product of two two-dimensional tori, $f: N \rightarrow N$ be a diffeomorphism, $f=\left(f_{1}, f_{2}\right)$, where $f_{i}: T^{2} \rightarrow T^{2}$ is a linear hyperbolic automorphism. Let $\lambda_{i}, \mu_{i}$ be the eigenvalues and $e_{i}, g_{i}$ be the corresponding eigenvectors, $i=1,2$. We assume that $0<\lambda_{1} \leq \lambda_{2}<1<\mu_{2} \leq \mu_{1}$. We take as the submanifold $M$ an open neighbourhood of the origin in the twodimensional plane spanned by the vectors $g_{1}, g_{2}$. Let $X_{1}$ (respectively $X_{2}$ ) be a segment on the straight line in the direction of the vector $g_{1}$ (respectively $g_{2}$ ). A simple calculation shows

$$
\operatorname{dim}_{(M, f)} X_{i}=\frac{2 \ln \mu_{i}}{\ln \mu_{1}+\ln \mu_{2}}, \quad i=1,2 .
$$

In particular,

$$
2>\operatorname{dim}_{(M, \cap)} X_{1} \geq 1 \geq \operatorname{dim}_{(M, \cap)} X_{2}>0 .
$$

We have at the same time that $\operatorname{dim}_{H} X_{1}=\operatorname{dim}_{H} X_{2}=1$. On the other hand if we take the straight line in the direction of the vector $g_{2}$ as the submanifold $M$ we have that

$$
\operatorname{dim}_{(M, f)} X_{1}=\operatorname{dim}_{H} X_{1}=1 \text {. }
$$

Remark. The systems $U_{f, \delta}$ have the property (P1) if the map $f$ uniformly expands (or contracts) on $X$. However one can give another definition of the dimension with respect to a map which will do for any map $f$. It will also coincide with the definition given above in the case when $f$ is an uniformly expanding (contracting) map. Let $X \subset M$ be a Borel subset. Define the outer measure on $X$ by the formula

$$
\bar{M}_{\alpha, \delta}(Y, X, M, f)=\lim _{n \rightarrow \infty} \inf _{\left\{U_{i}\right\} \in \bar{U}_{n, \delta}}\left\{\sum_{i} \nu\left(U_{i}\right)^{\alpha}: \bigcup_{i} U_{i} \supset Y\right\} .
$$

Here $Y \subset X$ is a subset and $\bar{U}_{n, \delta}, \delta>0, n \in \mathbb{Z}$ is a system of open sets $U$ for which there exist $m \in \mathbb{Z}$ and $x \in X$ such that $|m| \geq|n|$ and $U=U_{|m|}$ (cf. [11]). Essentially this approach means that in general we do not require the property (P1) to be satisfied. Now if we assume that the outer measures $\bar{m}_{\alpha, \delta}(Y, X, M, f)$ have the property (P2) we can define the dimension with respect to a map as specified above.

III. Let $\Lambda$ be a locally maximal hyperbolic set of a $C^{2}$-diffeomorphism $f: N \rightarrow N$ (cf. for example [13] in connection with definitions and results which we mention in this and subsequent sections). We assume that the map $f \mid \Lambda$ is topologically transitive. We denote by $W_{\text {loc }}^{s}(x)$ and $W_{\text {loc }}^{u}(x)$ (for $\left.x \in \Lambda\right)$ the local stable and unstable manifolds at the point $x$. Fix $x \in \Lambda$ and write $X=W_{\text {loc }}^{u}(x) \cap \Lambda$. Consider the family of systems $\bar{U}_{f, \delta}(X)$ given by formula (3) (where $M=W_{\mathrm{loc}}^{u}(x)$ ) and consider also the following families of systems:

$\bar{V}_{f, \delta}(X)=\left\{U \subset W_{\text {loc }}^{u}(x): U\right.$ is an open and connected set and there exist $n \in \mathbb{Z}$ and $x \in X$ such that

$$
f^{k}(U) \subset B_{W_{\mathrm{loc}}{ }^{\mu}\left(f^{k}(x)\right)}\left(f^{k}(x), \delta\right)
$$

for $k=0,1, \ldots, n-1$ and

$$
\left.f^{n}(U) \supset B_{w_{\text {loc }}^{u}\left(f^{n}(x)\right)}\left(f^{n}(x), \delta\right)\right\} .
$$


$\bar{V}_{f, \delta, C}(X)=\left\{U \subset W_{\mathrm{loc}}^{u}(x): U\right.$ is an open and connected set and there exist $n \in \mathbb{Z}$ and $x \in X$ such that

$$
f^{k}(U) \subset B_{W_{\mathrm{loc}}{ }^{k}\left(f^{k}(x)\right)}\left(f^{k}(x), C \delta\right)
$$

for $k=0,1, \ldots, n-1$ and

where $C \geq 1$ is a real number.

$$
\left.f^{n}(U) \supset B_{W_{\text {loc }}^{u}\left(f^{n}(x)\right)}\left(f^{n}(x), \delta\right)\right\},
$$

Denote by $E^{s}(x), E^{u}(x), x \in \Lambda$, the stable and unstable subspaces at the point $x$, and by $\chi^{i}(x)$ the values of the Lyapunov exponent at the point $x$. We assume that

$$
\chi^{1}(x) \geq \cdots \geq \chi^{k}(x)>0>\chi^{k+1}(x) \geq \cdots \geq \chi^{p}(x),
$$

where $k=\operatorname{dim} E^{s}(x)$ is independent of $x$. Denote by $\Gamma(f \mid \Lambda)$ the set of all Borel normalized $f$-invariant ergodic measures on $\Lambda$. We have that for $\mu \in \Gamma(f \mid \Lambda)$ and $\mu$-almost every $x \in \Lambda$

$$
\chi^{i}(x)=\chi_{\mu}^{i}=\text { const., } \quad i=1, \ldots, p
$$

Proposition 4. (1) For any $\delta>0$ small enough, the families $\bar{U}_{f, \delta}(X), \bar{V}_{f, \delta}(X)$, $\bar{V}_{f, \delta, C}(X), C \geq 1$, have the properties (P1) and (P2).

(2) The dimensions with respect to the map $f$ induced by outer measures $m_{\alpha}\left(Y, X, M, \bar{U}_{f, \delta}(X)\right), m_{\alpha}\left(Y, X, M, \bar{V}_{f, \delta}(X)\right), m_{\alpha}\left(Y, X, M, \bar{V}_{f, \delta, C}(X)\right)$ are equal and do not depend on the Riemannian metric on $\Lambda$.

We will assume that a Lyapunov metric is fixed on $\Lambda$.

Denote by $G_{\mu}$ the set of all $x \in \Lambda$ such that for any continuous function $\varphi$ on $\Lambda$ there exists the limit

$$
\lim _{n \rightarrow \infty} \frac{1}{n} \sum_{k=0}^{n-1} \varphi\left(f^{k}(x)\right)=\int_{\Lambda} \varphi(x) \mathrm{d} \mu(x) .
$$

Let $\varphi^{u}(x)=-\ln \mathrm{Jac}\left(d f \mid E^{u}(x)\right)$ and consider the pressure $P\left(t \varphi^{u}\right)$, corresponding to the function $t \varphi^{u}$, (cf. [4]), $0 \leq t \leq 1$. It is easy to prove the following statement.

Proposition 5. There exists a unique $t_{0} \in[0,1]$ such that $P\left(t_{0} \varphi^{u}\right)=0$.

We put for $x \in \Lambda$

$$
\begin{gathered}
Q_{\mu}(x)=\operatorname{dim}_{f}\left(W_{\text {loc }}^{u}(x) \cap G_{\mu}\right), \\
Q(x)=\operatorname{dim}_{f}\left(W_{\text {loc }}^{u}(x) \cap \Lambda\right) .
\end{gathered}
$$

The purpose of the present paper is to prove the following assertions.

THEOREM 1. Let $Z \subset \Lambda$ be an f-invariant Borel set such that $W_{\mathrm{loc}}^{s}(x) \cap \Lambda \subset Z$ for $x \in Z$. Then for every $x \in Z$ and any neighbourhood $U$ of $x$ in $W_{\mathrm{loc}}^{u}(x)$

$$
\operatorname{dim}_{f}(Z \cap U)=\text { const. }
$$

THEOREM 2. For any $x \in \Lambda$ and $\mu \in \Gamma(f \mid \Lambda)$.

$$
Q_{\mu}(x)=(p-k) h_{\mu}(f \mid \Lambda)\left(\left|\sum_{i=k+1}^{p} \chi_{\mu}^{i}\right|\right)^{-1}
$$

where $h_{\mu}(f \mid \Lambda)$ is the metric entropy of the map $f \mid \Lambda$. 
TheOrem 3. For any $x \in \Lambda$,

$$
Q(x)=\sup _{\mu \in \Gamma(f \mid \Lambda)} Q_{\mu}(x)=t_{0}(p-k) .
$$

COROLlARY. The following inequalities hold:

$$
\frac{-h(f)}{\int_{\Lambda} \varphi^{u} d m} \leq Q(x) \leq \frac{h(f)}{\left(h(f)-P\left(\varphi^{u}\right)\right)},
$$

where $h(f)$ denotes the topological entropy off and $m$ is the measure of maximal entropy.

Let $\Lambda$ be a locally maximal hyperbolic set for a diffeomorphism $f$. It is known that there is a neighbourhood $\mathcal{N} \subset \operatorname{Diff}^{1}(M)$ of $f$ such that any $g \in \mathcal{N}$ has a locally maximal hyperbolic set $\Lambda_{\mathrm{g}}$ near $\Lambda$. Let

$$
Q_{g}(x) \stackrel{\text { def }}{=} \operatorname{dim}_{g}\left(W_{\text {loc }}^{u}(x) \cap \Lambda_{g}\right) \text {. }
$$

It follows from theorem 3 that $Q_{g}(x)$ does not depend on $x$ but does depend on $g$.

THEOREM 4. The function $Q_{g}=Q_{g}(x)$ is continuous on the set $\mathcal{N}$.

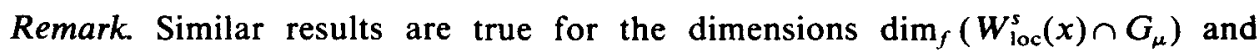
$\operatorname{dim}_{f}\left(W_{\text {loc }}^{s}(x) \cap \Lambda\right)$ with obvious modifications in the formulae.

If $\Lambda$ is a hyperbolic attractor for a diffeomorphism $f$ (cf. [1]), then

for any $x \in \Lambda$.

$$
\operatorname{dim}_{f}\left(W_{\text {loc }}^{u}(x) \cap \Lambda\right)=\operatorname{dim} W_{\text {loc }}^{u}(x)
$$

THEOREM 5. Let $\Lambda$ be a hyperbolic attractor for a diffeomorphism $f$. Then

$$
\operatorname{dim}_{f}\left(W_{\mathrm{loc}}^{s}(x) \cap \Lambda\right) \geq-k\left(\sum_{i=1}^{k} \chi_{\mu}^{i} / \sum_{i=k+1}^{p} \chi_{\mu}^{i}\right)
$$

where $\mu$ is the special measure constructed by Bowen, Ruelle and Sinai (cf. [5], [15]).

COROLlaRY. The following inequality holds:

$$
\sum_{i=1}^{p} x_{\mu}^{i} \leq 0
$$

This assertion follows immediately from theorem 5 .

We can consider these theorems as generalizations to the multi-dimensional case of the results obtained in the two-dimensional case in [11], [12].

\section{Proofs}

I. Proof of proposition 4. It is obvious that the systems $\bar{U}_{f, \delta}(X), \bar{V}_{f, \delta}(X), \bar{V}_{f, \delta, C}(X)$ have the property $(\mathrm{P} 1)$ with respect to $X$ (recall that we consider a Lyapunov metric on $\Lambda$ ). We will show that they have the property (P2). Take sufficiently small $\delta_{1} \leq \delta_{2}$. Let $U \in \bar{V}_{f, \delta_{1}}(X)$ have the form

$$
U=f^{-n}\left(B_{f^{n}(M)}\left(f^{n}(x), \delta_{1}\right)\right.
$$


where $M=W_{\text {loc }}^{u}(x)$ for some $x \in Y, n \geq 0$. Consider the set

$$
\Psi(U)=f^{-n}\left(B_{f^{n}(M)}\left(f^{n}(x), \delta_{2}\right)\right) \in \bar{V}_{f, \delta_{2}}(X) .
$$

It is easy to see that

$$
\bar{U} \subset \Psi(U), \quad \operatorname{diam} U \leq C_{1} \operatorname{diam} U_{2}, \quad \nu(\Psi(U)) \leq C_{2} \nu(U),
$$

where $C_{1} \geq 1, C_{2} \geq 1$ are constants depending only on $\delta_{1}, \delta_{2}$. It follows from (6) that for any $\varepsilon>0$ and $Y \subset X$

$$
M_{\alpha, \varepsilon}\left(Y, X, M, \bar{U}_{f, \delta_{1}}(X)\right) \geq \frac{1}{C_{2}^{\alpha}} M_{\alpha, C_{1} \varepsilon}\left(Y, X, M, \bar{U}_{f, \delta_{1}}(X)\right)
$$

We have from this that

$$
\begin{aligned}
m_{\alpha}\left(Y, X, M, \bar{U}_{f, \delta_{1}}(X)\right) & \geq \frac{1}{C_{2}^{\alpha}} m_{\alpha}\left(Y, X, M, \bar{U}_{f, \delta_{2}}(X)\right) \\
& \geq \frac{1}{C_{2}} m_{\alpha}\left(Y, X, M, \bar{U}_{f, \delta_{2}}(X)\right)
\end{aligned}
$$

Now let $U \in \bar{U}_{f, \delta_{2}}(X)$ have the form

$$
U=f^{-n}\left(B_{f^{n}(M)}\left(f^{n}(x), \delta_{2}\right)\right.
$$

for some $x \in Y, n \geq 0$. There exists $N=N\left(\delta_{1}, \delta_{2}\right)$ and points $x_{1}, \ldots, x_{N} \in U \cap Y$ such that

$$
\bigcup_{i=1}^{N} B_{f^{n}(M)}\left(f^{n}\left(x_{i}\right), \delta_{1}\right) \supset B_{f^{n}(M)}\left(f^{n}(x), \delta_{2}\right) \cap f^{n}(Y) .
$$

We will write $U_{i}$ for $f^{-n}\left(B_{f^{n}(M)}\left(f^{n}\left(x_{i}\right), \delta_{1}\right)\right.$. It is easy to see that we have, for sufficiently small $\delta_{1}, \delta_{2}$,

$$
\begin{gathered}
\bigcup_{i=1}^{N} U_{i} \supset U \cap Y, \quad \sum_{i=1}^{N} \nu\left(U_{i}\right)^{\alpha} \leq N \nu(U)^{\alpha}, \\
\operatorname{diam} U_{i} \leq \operatorname{diam} U .
\end{gathered}
$$

Therefore

$$
M_{\alpha, \varepsilon}\left(Y, X, M, \bar{U}_{f, \delta_{2}}(X)\right) \geq(1 / N) M_{\alpha, \varepsilon}\left(Y, X, M, \bar{U}_{f, \delta_{1}}(X)\right) .
$$

Taking the limit as $\varepsilon \rightarrow 0$ we get

$$
m_{\alpha}\left(Y, X, M, \bar{U}_{f, \delta_{2}}(X)\right) \geq(1 / N) m_{\alpha}\left(Y, X, M, \bar{U}_{f, \delta_{2}}(X)\right)
$$

The condition (P2) follows from here and inequality (7). We can show in the same way that for any $\varepsilon>0$ and $Y \subset X$

$$
\begin{aligned}
M_{\alpha, \varepsilon}\left(Y, X, M, \bar{U}_{f, \delta}(X)\right) & \geq M_{\alpha, \varepsilon}\left(Y, X, M, \bar{V}_{f, \delta}(X)\right) \\
& \geq \frac{1}{C_{2}^{\alpha}} M_{\alpha, \varepsilon}\left(Y, X, M, \bar{U}_{f, C_{3} \delta}(X)\right),
\end{aligned}
$$

where $C_{3} \geq 1$ is some constant independent of $\varepsilon, \delta$. Thus we have the condition (P2) for the system $\bar{V}_{f, \delta}(X)$, and equality for the dimensions with respect to the map $f$, induced by the systems $\bar{U}_{f, \delta}(X)$ and $\bar{V}_{f, \delta}(X)$. Repeating the arguments given above for the system $\bar{V}_{f, \delta, C}(X)$ we complete the proof of proposition 4. 
II. Proof of theorem 1. Let $\mathscr{A}$ be a Markov partition consisting of the open rectangles $\Pi_{1}, \ldots, \Pi_{n}$ such that

$$
\prod_{i}=\left(U_{i} \cap \Lambda\right) \times\left(V_{i} \cap \Lambda\right)
$$

where $U_{i}$ and $V_{i}$ are open sets in the submanifolds $W_{\text {loc }}^{s}\left(x_{i}\right)$ and $W_{\text {loc }}^{u}\left(x_{i}\right)$ respectively (cf. [4]). Let

$$
A(x)=W_{\text {loc }}^{u}(x) \cap \prod_{i(x)}, \quad x \in \Lambda,
$$

where $i(x)$ is the number of rectangles containing the point $x$.

Lemma 1. Let $y_{1}, y_{2} \in \prod_{i}$ for some $i$. Then

$$
\operatorname{dim}_{f}\left(A\left(y_{1}\right) \cap Z\right)=\operatorname{dim}_{f}\left(A\left(y_{2}\right) \cap Z\right) .
$$

Proof. Suppose for $\delta>0$ and $i=1,2$.

$$
\bar{V}_{i}=\bar{U}_{f, \delta}\left(A\left(y_{i}\right) \cap Z\right)
$$

(cf. [11]). Let $U \in \bar{V}_{1}$ have the form

$$
U=f^{-n}\left(B_{f^{n}\left(w_{\text {loc }}^{u}\left(y_{1}\right)\right)}\left(f^{n}(z), \delta\right)\right)
$$

for some $n>0$ and $z \in A\left(y_{1}\right) \cap Z$. We put

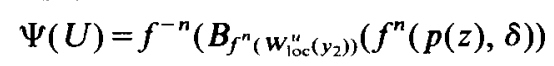

where $p: A\left(y_{1}\right) \rightarrow A\left(y_{2}\right)$ is the Poincare map given by the local manifolds $W_{\text {loc }}^{s}(z)$. We note that $p(z) \in A\left(y_{2}\right) \cap Z$ by virtue of our conditions on $Z$. Therefore $\Psi(U) \in \bar{V}_{2}$. It follows from the results of [4] that for any $U \in \bar{V}_{1}$

$$
C^{-1} \nu(U) \leq \nu(\Psi(U)) \leq C \nu(U) \text {. }
$$

As $\Psi$ is a one-to-one map the systems $\bar{V}_{1}$ and $\bar{V}_{2}$ are equivalent. Therefore the required result follows from propositions 2 and 3.

Lemma 2. Let $y_{1}, y_{2} \in \Lambda$. Then

$$
\operatorname{dim}_{f}\left(A\left(y_{1}\right) \cap Z\right)=\operatorname{dim}_{f}\left(A\left(y_{2}\right) \cap Z\right) .
$$

Proof. As $\mathscr{A}$ is a Markov partition there exists $m>0$ such that

for some $z \in \prod_{i\left(y_{2}\right)}$.

$$
f^{m}\left(A\left(y_{1}\right)\right) \cap A\left(y_{2}\right)=A(z) \neq \varnothing
$$

It is easy to see that systems $\bar{U}_{f, \delta}(A(z) \cap Z)$ and $\bar{U}_{f, \delta}\left(f^{-m}(A(z)) \cap Z\right)$ are equivalent because $f$ is a diffeomorphism and $Z$ is an $f$-invariant set. Now propositions 2 and 3 and lemma 1 imply that

$$
\begin{aligned}
\operatorname{dim}\left(A\left(y_{1}\right) \cap Z\right) & \geq \operatorname{dim}_{f}\left(f^{-m}(A(z)) \cap Z\right) \\
& =\operatorname{dim}_{f}(A(z) \cap Z) \\
& =\operatorname{dim}_{f}\left(A\left(y_{2}\right) \cap Z\right) .
\end{aligned}
$$

The opposite inequality is proved in the same way; the lemma is now proved.

Let $y_{1}, y_{2} \in \Lambda, U_{1}, U_{2}$ be neighbourhoods of points $y_{1}$ and $y_{2}$ in $W_{\text {loc }}^{u}\left(y_{1}\right)$ and $W_{\text {loc }}^{u}\left(y_{2}\right)$. If $a=\max _{i} \operatorname{diam} \prod_{i}$ is sufficiently small then $U_{1} \supset \boldsymbol{A}\left(y_{1}\right)$. On the other hand there exist $z_{1}, \ldots, z_{s} \in W_{\text {loc }}^{u}\left(y_{2}\right)$ such that $U_{2} \subset \bigcup_{i=1}^{s} A\left(z_{i}\right)$. Therefore it follows from proposition 3 and lemma 2 that

$$
\operatorname{dim}_{f}\left(U_{1} \cap Z\right) \geq \operatorname{dim}_{f}\left(A\left(y_{1}\right) \cap Z\right) \geq \operatorname{dim}_{f}\left(U_{2} \cap Z\right) .
$$

The opposite inequality is proved in the same way, so the theorem is proved. 
III. Proof of proposition 5 (cf. [11], [4]). It follows directly from the definition of the pressure (cf. [4]) that

$$
P\left(t_{1} \varphi^{u}\right)<P\left(t_{2} \varphi^{u}\right) \quad \text { if } t_{2}<t_{1}
$$

It is also obvious that $P(0) \geq 0$. On the other hand it is known that $P\left(\varphi^{u}\right) \leq 0$ (cf. [4]). The result we need follows from here.

IV. The proof of theorem 2 is a modification of the arguments given in [12].

LEMMA 3. There exist $C>0$, a sequence of numbers $\delta_{n} \downarrow 0$, and a sequence of covers $\mathscr{A}_{n}=\left\{\prod_{1}^{(n)}, \ldots, \prod_{k_{n}}^{(n)}\right\}$ of the set $\Lambda$ by open rectangles $\prod_{i}^{(n)}$ such that:

(1) $a_{n} \stackrel{\text { def }}{=} \max _{1 \leq i \leq k_{n}} \operatorname{diam} \prod_{i}^{(n)} \rightarrow 0$ as $n \rightarrow \infty$;

(2) for every $x \in \prod_{i}^{(n)}$, there is an open subset $U_{i, n}^{u}(x)$ in $W_{\mathrm{loc}}^{u}(x)$ having the following properties:

(a) for any $y \in W_{\mathrm{loc}}^{u}(x) \cap \prod_{i}^{(n)}, U_{i, n}^{u}(x)=U_{i, n}^{u}(y)$;

(b) $B_{W_{\text {loc }}^{u}(x)}\left(x, C \delta_{n}\right) \supset U_{i, n}^{u}(x) \supset B_{W_{\text {loc }}^{u}(x)}\left(x, \delta_{n}\right)$.

The proof follows easily from the continuity of local stable and unstable manifolds on the set $\Lambda$. As 'the size' of an unstable manifold is in general a Hölder function on $\Lambda$ then the diameters of the sets $U_{i, n}^{u}(x)$ are 'much bigger' than 'the sizes' of the rectangles $\prod_{i}^{(n)}$ in the direction of the stable manifolds.

Remark. We can assume that $\delta_{1} \leq \delta_{0}$ (cf. the condition (P2)).

Write

$$
\tilde{\Pi}_{i}^{(n)}=\bigcup_{x \in \prod_{i}^{(n)}} U_{i, n}^{u}(x)
$$

Fix $\varepsilon>0$ and take $n>0$ so large that for any $i=1, \ldots, k_{n}, y_{1}, y_{2} \in \prod_{i}^{(n)}$

$$
\left|\varphi^{u}\left(y_{1}\right)-\varphi^{u}\left(y_{2}\right)\right| \leq \varepsilon
$$

It is shown in [3] that

$$
h_{\mu}(f \mid \Lambda)=h\left(f, G_{\mu}\right) \stackrel{\text { def }}{=} h
$$

where $h\left(f, G_{\mu}\right)$ is the topological entropy on the non-compact set $G_{\mu}$. Using the definition of topological entropy given in [3] we have that for any $\gamma>0$ there exists a cover $\left\{Q_{i}^{(n)}\right\}$ of the set $G_{\mu}\left(Q_{i}^{(n)}\right.$ are open subsets in $\left.\Lambda\right)$ such that

$$
D\left(Q_{i}^{(n)}\right) \stackrel{\text { def }}{=} \sum_{Q_{i}^{(n)}} \exp \left(-n\left(Q_{i}^{(n)}\right)(h+\varepsilon)\right) \leq \gamma
$$

Here $n\left(Q_{i}^{(n)}\right)$ is the largest integer having the following property: $f^{k}\left(Q_{i}^{(n)}\right)$ is contained in some element of the covering $\mathscr{A}_{n}$ for $k=0,1, \ldots, n\left(Q_{i}^{(n)}\right)$.

Fix $x_{0} \in \Lambda$ and consider a $Q_{i}^{(n)}$ such that $W_{\text {loc }}^{u}(x) \cap Q_{i}^{(n)} \neq \varnothing$. Let $x \in W_{\text {loc }}^{u}\left(x_{0}\right) \cap Q_{i}^{(n)}$. Take the integer $j$ such that

$$
f^{-n\left(Q_{i}^{(n)}\right.}\left(Q_{i}^{(n)}\right) \subset \prod_{j}^{(n)}
$$

and denote by

$$
V_{i}^{(n)}(x)=f^{-n\left(Q_{i}^{(n)}\right)}\left(U_{j, n}^{u}\left(f^{n\left(Q_{i}^{(n)}\right.}(x)\right)\right.
$$


It is easy to see that

$$
V_{i}^{(n)}(x) \supset Q_{i}^{(n)} \cap W_{\text {loc }}^{u}\left(x_{0}\right) .
$$

In addition we have from lemma 3 that

$$
V_{i}^{(n)}(x) \in \bar{V}_{f, \delta_{n}, C}\left(W_{\mathrm{loc}}^{u}(x) \cap G_{\mu}\right)
$$

Let

$$
G_{\mu, r}=\left\{x \in G_{\mu}:\left|\frac{1}{m} \sum_{i=0}^{m-1} \varphi^{u}\left(f^{i}(x)\right)+\int_{\Lambda} \varphi^{u} d \mu\right| \leq \varepsilon \quad \text { for any } m \geq r\right\} .
$$

Notice that $G_{\mu}=\bigcup_{r} G_{\mu, r}$ and

$$
\chi \stackrel{\text { def }}{=} \sum_{i=k+1}^{p} \chi_{\mu}^{i}=-\int_{\Lambda} \varphi^{u} d \mu,
$$

because $\mu$ is an ergodic measure. We will give an estimate from above of $\operatorname{dim}_{f}\left(G_{\mu, r} \cap\right.$ $\left.W_{\text {loc }}^{u}(x)\right)$, independent of $r$. If $\gamma$ is sufficiently small then by virtue of $(12), n\left(Q_{i}^{(n)}\right) \geq r$ for each $i=1, \ldots, k_{n}$. Therefore it follows from the definition of $G_{\mu, r}$, inequality (10) and equalities (13) and (14) that for any $x \in G_{\mu, r}$,

$$
\begin{aligned}
\nu\left(V_{i}^{(n)}(x)\right)= & \int_{U_{i, n}^{u}\left(f^{\left.m\left(Q_{i}^{(n)}\right)(x)\right)}\right.} \operatorname{Jac}\left(d f^{-n\left(Q_{i}^{(n)}\right)}(y) d \nu(y)\right. \\
= & \operatorname{Jac}\left(d f^{-n\left(Q_{i}^{(n)}\right)}(x)\right) \int_{U_{j, n}^{u}\left(f^{n \prime \prime} Q_{i}^{(n)}(x)\right)} \operatorname{Jac}\left(d f^{-n\left(Q_{i}^{(n)}\right)}(y)\right) \\
& \times\left[\operatorname{Jac}\left(d f^{-n\left(Q_{i}^{(n)}\right)}(x)\right)\right]^{-1} d \nu(y) \\
\leq & \exp \left[(-\chi+\varepsilon) n\left(Q_{i}^{(n)}\right)\right] \times \exp \left[\varepsilon n\left(Q_{i}^{(n)}\right)\right] \\
& \times \nu\left(U_{j, n}^{u}\left(f^{n\left(Q_{i}^{(n)}\right.}(x)\right)\right) \\
\leq & C a_{n} \exp (-\chi+2 \varepsilon) n\left(Q_{i}^{(n)}\right) .
\end{aligned}
$$

Then for any $\tau>0$ we have diam $V_{i}^{(n)}(x) \leq \tau$ if $x \in G_{\mu}$ and $r$ is large enough (and consequently $n\left(Q_{i}^{(n)}\right)$ is also large enough). Using (11), (12), (15) we find that

$$
\sum \nu\left(V_{i}^{(n)}(x)\right)^{(h+\varepsilon) /(x-2 \varepsilon)} \leq \gamma\left(C a_{n}\right)^{(h+\varepsilon) /(x-2 \varepsilon)}
$$

is arbitrarily small. As $\varepsilon$ is arbitrarily small we have from proposition 3 that

$$
\operatorname{dim}_{f}\left(W_{\mathrm{loc}}^{u}(x) \cap G_{\mu}\right)=\sup _{r} \operatorname{dim}_{f}\left(W_{\mathrm{loc}}^{u}(x) \cap G_{\mu, r}\right) \leq(h / \chi)(p-k) .
$$

Now we will prove the estimation from below. For given $\varepsilon>0$ let $\mathscr{A}$ be a cover of $\Lambda$ consisting of open rectangles $\prod_{i}$ such that

$$
\left|\varphi^{u}(x)-\varphi^{u}(y)\right| \leq \varepsilon,
$$

for any $x, y \in \prod_{i}$. There exists $\delta_{1}>0$ having the following property: let $U \subset \Lambda$ be a set such that if the diameters of the sets $W_{\text {loc }}^{u}(x) \cap U$ and $W_{\text {loc }}^{s}(x) \cap U$ are less than $\delta_{1}$ for any $x \in \Lambda$, then $U$ is contained in some element of the covering $\mathscr{A}$. We can assume that $\delta_{1} \leq \delta_{0}$ (cf. the condition (P2)). Take any $x \in \Lambda$ and choose $m>0$ so large that for every $y \in \Lambda$

$$
f^{m}\left(W_{\mathrm{loc}}^{u}(x)\right) \cap B_{W_{\text {loc }}^{s}(y)}\left(y, \delta_{1}\right) \neq \varnothing .
$$


It is easy to see that for any $r>0$

$$
\begin{aligned}
\operatorname{dim}_{f}\left(G_{\mu, r} \cap f^{m}\left(W_{\mathrm{loc}}^{u}(x)\right)\right) & =\operatorname{dim}_{f}\left(G_{\mu} \cap f^{m}\left(W_{\mathrm{loc}}^{u}(x)\right)\right) \\
& =\operatorname{dim}_{f}\left(G_{\mu} \cap W_{\mathrm{loc}}^{u}(x)\right) \stackrel{\text { def }}{=} d .
\end{aligned}
$$

Therefore, by proposition 4 , for any $\alpha>0$ and any sufficiently small $\tau>0$ there exists a cover of the set

$$
X \stackrel{\text { def }}{=} G_{\mu, r} \cap f^{m}\left(W_{\text {loc }}^{u}(x)\right)
$$

by the sets $\left\{V_{i}\right\}$ such that $\operatorname{diam} V_{i} \leq \tau, V_{i} \in U_{f, \delta}(X)$, (here $\delta=\delta_{1} / 2$ ), and

$$
\sum_{i} \nu\left(V_{i}\right)^{d+\varepsilon} \leq \alpha
$$

It follows from the definition of the system $\bar{U}_{f, \delta}(X)$ that for any $i$ there exist $n_{i}$ and $y_{i} \in X$ such that

$$
V_{i}=f^{-n_{i}}\left(B_{W_{\mathrm{loc}}^{u}\left(f^{\left.n_{i}\left(y_{i}\right)\right)}\right.}\left(f^{n_{i}}\left(y_{i}\right), \delta\right)\right)
$$

Let

$$
U_{i}=\left(\bigcup_{y \in V, \cap \Lambda} B_{W_{\mathrm{loc}}^{s}(y)}(y, \delta)\right) \cap \Lambda
$$

It follows from (16) that $\bigcup_{i} U_{i} \supset G_{\mu, r}$. In addition

$$
n\left(U_{i}\right) \geq n_{i}
$$

If $\tau$ is small enough then all $n_{i}>r$ and we have

$$
K \delta^{p-k} \leq \nu\left(V_{i}\right) \exp \left[(\chi+2 \varepsilon) n_{i}\right]
$$

where $K>0$ is some constant; (recall that $p-k=\operatorname{dim} E^{u}(x)$ ). Therefore by virtue of (17) and (18)

$$
\begin{aligned}
& \left.\sum \exp (-(d+\varepsilon)(\chi+2 \varepsilon)) n\left(V_{i}\right)\right) \\
& \quad \leq K \delta^{-(p-k)(d+\varepsilon)} \sum \nu\left(V_{i}\right)^{d+\varepsilon} \leq \alpha K \delta^{-(p-k)(d+\varepsilon)} .
\end{aligned}
$$

Now the proof is completed by means of the arguments given in [12].

V. Proof of theorem 3. Denote by $\mu_{0}$ the Gibbs measure corresponding to the function $t_{0} \varphi^{u}$. It is known (cf. [4]) that

$$
h_{\mu_{0}}(f \mid \Lambda)=t_{0} \int_{\Lambda} \varphi^{u} d \mu_{0}=t_{0} \sum_{i=k+1}^{p} \chi_{\mu}^{i} .
$$

It follows from here, proposition 3 and theorem 2 that

$$
Q(x) \geq Q_{\mu_{0}}(x)=(p-k) t_{0} .
$$

We will now prove the opposite inequality. The following assertion is an easy modification of lemma 1.

LEMMA 4. There exist $l>0, C>0$, a sequence of numbers $\delta_{n} \downarrow 0$ and a sequence of covers $\mathscr{A}_{n}=\left\{\prod_{1}^{(n)}, \ldots, \prod_{k_{n}}^{(n)}\right\}$ of the set $\Lambda$ by open rectangles $\prod_{i}^{(n)}$ such that:

(1) $a_{n} \stackrel{\text { def }}{=} \max _{1 \leq i \leq k_{n}} \operatorname{diam} \prod_{i}^{(n)} \rightarrow 0$ as $n \rightarrow \infty$; 
(2) for any $x \in \prod_{i}^{(n)}$ there is an open subset $U_{i, n}^{u}(x)$ in $W_{\text {loc }}^{u}(x)$ having the following properties:

(a) for any $y \in W_{\text {loc }}^{u}(x) \cap \prod_{i}^{(n)}$,

$$
U_{i, n}^{u}(y)=U_{i, n}^{u}(x)
$$

(b) $B_{W_{\mathrm{loc}(x)}^{u}}\left(x, C \delta_{n}\right) \supset U_{i, n}^{u}(x) \supset B_{W_{\mathrm{loc}}^{u}(x)}\left(x, \delta_{n}\right)$;

(3) for any $x \in \Lambda$, any rectangle $\prod_{i}^{(n)}$ containing the point $x$, and any rectangle $\prod_{j}^{(n)}$ containing the point $f^{-1}(x)$, we have

$$
f^{-l}\left(U_{i, n}^{u}(x)\right) \subset U_{j, n}^{u}\left(f^{-l}(x)\right) .
$$

We can assume that $\delta_{1} \leq \delta_{0}$ (cf. the condition (P2)). Let $F=f^{l}$ and

$$
\bar{Q}(x)=\operatorname{dim}_{F}\left(W_{\text {loc }}^{u}(x) \cap \Lambda\right) \text {. }
$$

It follows from the definition of the dimension with respect to a map that $\bar{Q}(x)=Q(x)$. To prove the estimation from above we must show that

$$
\bar{Q}(x) \leq(p-k) t_{0} \text {. }
$$

We have from the definition of the pressure that $t_{0}$ is a root of the equation

$$
P_{F}\left(t \varphi_{F}^{u}\right)=0 \text {, }
$$

where $\varphi_{F}^{u}(x)=-\ln \operatorname{Jac}\left(F \mid E^{u}(x)\right) \mid$. If $t_{0}=1$ then our statement follows from proposition 3. Therefore we can assume that $0 \leq t_{0}<1$. This means that for any $\varepsilon>0$ small enough

$$
P=P\left(\left(t_{0}+\varepsilon\right) \varphi_{F}^{u}\right)<0 .
$$

Fix a sufficiently small $\varepsilon>0$ and a sufficiently large $n>0$. According to the definition of the pressure given in [4] we consider for a given $m>0$, the set $W_{m}\left(\mathscr{A}_{n}\right)$ consisting of all collections of length $m$, made up of the elements of the cover $\mathscr{A}_{n} ; \Pi_{-}^{(n)}$ $=\prod_{i_{0}}^{(n)} \prod_{i_{1}}^{(n)} \cdots \prod_{i_{m-1}}^{(n)} \in W_{m}\left(\mathscr{A}_{n}\right)$. Let

$$
\begin{aligned}
X\left(\prod_{-}^{(n)}\right) & =\left\{x \in \Lambda: F^{k}(x) \in \prod_{i_{k}}^{(n)} \quad \text { for } k=0, \ldots, m-1\right\}, \\
S_{m}\left(\Pi_{-}^{(n)}\right) & =\left(t_{0}+\varepsilon\right) \sup \left\{\sum_{k=0}^{m-1} \varphi^{u}\left(F^{k}(x)\right): x \in X\left(\prod_{-}^{(n)}\right)\right\} .
\end{aligned}
$$

If $X\left(\Pi_{-}^{(n)}\right)=\varnothing$ then we put $S_{m}\left(\Pi_{-}{ }^{(n)}\right)=-\infty$. We say that $\Gamma \subset W_{m}\left(\mathscr{A}_{n}\right)$ covers $\Lambda$ if $\Lambda=\bigcup_{\Pi^{(n)} \in \Gamma} X\left(\Pi^{(n)}\right)$. We put

$$
Z_{m}\left(\mathscr{A}_{n}\right)=\inf _{\Gamma} \sum_{\Gamma_{-}^{(n)} \in \Gamma} \exp S_{m}\left(\Pi_{-}^{(n)}\right)
$$

where $\Gamma$ runs through all subsets of $W_{m}\left(\mathscr{A}_{n}\right)$ covering the set $\Lambda$. Then

$$
P=\lim _{\operatorname{diam} \mathscr{A}_{n} \rightarrow 0} \lim _{m \rightarrow \infty} \frac{1}{m} \ln Z_{m}\left(\mathscr{A}_{n}\right)
$$

It follows from (19) that for any $\gamma>0$ and any sufficiently large $n$ and $m$

We take $\gamma$ so small that

$$
Z_{m}\left(\mathscr{A}_{n}\right) \leq \exp (P+2 \gamma) m \text {. }
$$

$$
P+4 \gamma<0
$$


Let $\Gamma$ be a subset in $W_{m}\left(\mathscr{A}_{n}\right)$ covering $\Lambda$ and such that

$$
\sum_{\Pi^{(n)} \in \Gamma} \exp S_{m}\left(\Pi_{-}^{(n)}\right) \leq \exp (P+3 \gamma) m .
$$

Consider the rectangle $\prod_{1}^{(n)}$ and the point $x \in \prod_{1}^{(n)}$. By theorem 1 it is sufficient to prove that

$$
\operatorname{dim}_{F}\left(\prod_{1}^{(n)} \cap W_{\text {loc }}^{u}(x)\right) \leq t_{0}(p-k) .
$$

Denote by $\Gamma^{l}$ the set of all collections $\Pi_{-}^{(n)} \in \Gamma$ which have $\prod_{1}^{(n)}$ as the first element. It is easy to see that

(1) $X\left(\Pi_{-}^{(n)}\right)$ is an open subset in $\prod_{1}^{(n)}$;

(2) $\bigcup_{\Pi^{(n)} \in \Gamma^{\prime}} X\left(\prod_{-}^{(n)}\right) \supset \prod_{1}^{(n)}$.

Consider the rectangles $\tilde{\Pi}_{i}^{(n)}$ defined using the rectangles $\prod_{i}^{(n)}, i=1, \ldots, k_{n}$ in (9). We put for $\Pi_{-}^{(n)}=\prod_{1}^{(n)} \prod_{i_{1}}^{(n)} \cdots \prod_{i_{m-1}}^{(n)} \in \Gamma^{1}$ :

$$
\begin{aligned}
& \tilde{Y}\left(\Pi_{-}^{(n)}\right)=\bigcap_{k=1}^{m-1} F^{-k}\left(\tilde{\Pi}_{i_{k}}^{(n)}\right) \cap \tilde{\Pi}_{1}^{(n)}, \\
& Y\left(\Pi_{-}^{(n)}\right)=\tilde{Y}\left(\Pi_{-}^{(n)}\right) \cap W_{\text {loc }}^{u}(x) .
\end{aligned}
$$

The following properties of the sets $Y\left(\Pi_{-}^{(n)}\right)$ are consequences of the construction given above, properties (1), (2) of the sets $X\left(\Pi_{-}^{(n)}\right)$, and lemma 2:

(1) $Y\left(\Pi_{-}^{(n)}\right)$ is an open subset of $W_{\mathrm{loc}}^{u}(x)$;

(2) $\bigcup_{\Pi^{(n)} \in \Gamma^{1}} Y\left(\Pi_{-}^{(n)}\right) \supset \prod_{1}^{(n)} \bigcap W_{\text {loc }}^{u}(x)$;

(3) $Y\left(\prod_{-}^{(n)}\right) \in \bar{V}_{F, \delta_{n}, C}$.

We have for any $\gamma>0$ and sufficiently large $n$, that for every $y_{1}, y_{2} \in \prod_{i}^{(n)}$

$$
\left|\varphi^{u}\left(y_{1}\right)-\varphi^{u}\left(y_{2}\right)\right| \leq \gamma \text {. }
$$

Therefore

$$
\begin{aligned}
D \stackrel{\text { def }}{=} & \sum_{\Gamma^{(n)} \in \Gamma^{\prime}} \nu\left(Y\left(\left.\Pi_{-}\right|^{(n)}\right)\right)^{t_{0}+\varepsilon} \\
\leq & \left(C \delta_{n}\right)^{p-k} \sum_{\Pi^{(n)} \in \Gamma^{1}} \sup _{y \in Y\left(\Pi_{-}^{(n)}\right)}\left[\operatorname{Jac}\left(F^{k} \mid T_{y} W_{\mathrm{loc}}^{u}(x)\right]^{-\left(t_{0}+\varepsilon\right)}\right. \\
\leq & \left(C \delta_{n}\right)^{p-k} \exp \left((\gamma m)\left(t_{0}+\varepsilon\right)\right) \\
& \times \sum_{\Gamma_{-}^{(n)} \in \Gamma^{1}} \sup _{y \in X\left(\Pi^{(k)}\right)}\left[\operatorname{Jac}\left(F^{k} \mid E^{u}(y)\right)\right]^{-\left(t_{0}+\varepsilon\right)} \\
\leq & \left(C \delta_{n}\right)^{p-k} \exp \left(\gamma m\left(t_{0}+\varepsilon\right)\right) \sum_{\Pi^{(n)} \in \Gamma^{1}} \exp S_{m}\left(\Pi_{-}^{(n)}\right) .
\end{aligned}
$$

It follows from here, the definitions of the function $\varphi^{u}$ and the sets $Z_{m}\left(\mathscr{A}_{n}\right)$ and inequality (22) that

$$
D \leq\left(C \delta_{n}\right)^{p-k} \exp (P+4 \gamma) m
$$

Therefore if $m$ is sufficiently large then $D$ is arbitrarily small by (17). This fact proves (18) and consequently the statement we need.

VI. The proof of theorem 5 is an easy modification of theorem 2 . The proofs of the corollary and theorem 4 repeat the arguments given in [11]. 
I would like to thank D. V. Anosov for his help and for useful discussions during the preparation of this paper; I would also like to thank Ya. G. Sinai for his attention to this paper.

\section{REFERENCES}

[1] V. A. Afraimovich \& Ya. B. Pesin. Estimations of the dimension of a hyperbolic set in the neighbourhood of a homoclinic point. Russian Math. Surveys, 1984. To appear.

[2] P. Billingsley. Ergodic Theory and Information Wiley: New York 1965.

[3] R. Bowen. Topological entropy for noncompact sets. Trans. Amer. Math. Soc. 184 (1973), 125-136.

[4] R. Bowen. Equilibrium States and the Ergodic Theory of Anosov Diffeomorphisms. Lecture Notes in Maths 470, Springer: Berlin, 1975

[5] R. Bowen \& D. Rouelle. The ergodic theory of Axiom A flows. Invent Math. 29 (1975), 181-202.

[6] A. Douady \& J. Oesterlé. Dimension de Hausdorff des attracteures. C.R. Acad. Sci. Paris, 24 (1980), 1135-1138.

[7] F. Federer, Geometric Measure Theory. Springer-Verlag: Berlin, 1969.

[8] W. Hurewicz \& H. Wallman. Dimension Theory. Princeton Univ. Press: Princeton, 1941.

[9] A. Katok. Lyapunov exponents, entropy and periodic orbits for diffeomorphisms. Publ. Math. IHES, 51 (1980), 137-170.

[10] F. Ledrappier. Some relations between dimension and Lyapunov exponents. Comm. Math. Phys., 81 (1981), 229-238.

[11] H. McCluskey \& A. Manning. Hausdorff dimension for horseshoes. Ergod. Th. \& Dynam. Sys. 3 (1983), 251-260.

[12] A. Manning. A relation between Lyapunov exponents, Hausdorff dimension and entropy. Ergod. Th. \& Dynam. Sys. 1 (1981), 451-459.

[13] Ya. B. Pesin \& Ya. G. Sinai. Hyperbolicity and Stochasticity of Dynamical Systems. Math.-Phys. Review 2, 53-116; Harwood Acad. Publ. GMBH.

[14] D. Ruelle \& F. Takens. On the nature of turbulence. Comm. Math. Phys. 20 (1971), 167-192.

[15] Ya. G. Sinai. Gibbs measures in ergodic theory. Russian Math. Surveys 27 (1972), $21-64$.

[16] F. Takens. Detecting strange attractors in turbulence. Springer Lect. Notes in Math, 898 (1981), 366-381.

[17] L.-S. Young. Capacity of attractors. Ergod. Th. \& Dynam. Syst. 1 (1981), 381-388. 